where $\psi$ is a solution of

$$
\psi^{\prime \prime}+\alpha \nu(1+\nu) \psi=\varphi_{*}^{\prime \prime} .
$$

Equation (5.6) can be solved by Latta's method if $\psi$ (and, therefore, $\varphi$ ) is an exponential polynomial. The details of the computations, though complicated, are not impossible, but it would serve no purpose to complete them here.

\title{
ReFerences
}

1. J. Halcombe Laning, Jr. and Richard H. Battin, Random processes in automatic control, McGrawHill, 1956

2. Harold Levine, Diffraction by an infinite slit, Stanford Univ. Appl. Math. and Stat. Lab. Tech. Rept. no. 61 (1957)

3. C. J. Tranter, $A$ further note on dual integral equations and an application to the diffraction of electromagnetic waves, Quart. J. Mech. and Appl. Math. VII, part 3, 317-325 (1954)

4. Gordon Latta, The solution of a class of integral equations reducible to ordinary differential equations, Stanford Univ. Appl. Math. and Stat. Lab. Tech. Rept., no. 32 (1955)

5. G. E. Latta, The solution of a class of integral equations, J. Ratl. Mech. and Anal. 5, no 5, 821-833 (1956)

6. Carl E. Pearson, On the finite strip problem, Quart. Appl. Math. XV, no 2, 202-208 (1957)

7. T. Carlemann, Uber die Abelsche Integralgleichung mit konstanten Integrationsgrenzen, Math. Z. 15 111-120 (1922)

\section{ON THE FIRST STABILITY INTERVAL OF THE HILL EQUATION*}

BY C. R. PUTNAM (Purdue University)

Let $\lambda$ denote a real parameter and let $f=f(t)$ be a real-valued, continuous periodic function of period 1. It is known (Liapounoff) that the Hill equation

$$
x^{\prime \prime}+(\lambda+f(t)) x=0 \quad\left[{ }^{\prime}=d / d t, f(t+1)=f(t)\right]
$$

is stable for $\lambda=0$, so that every solution of the equation $x^{\prime \prime}+f(t) x=0$ is bounded, whenever

$$
f \geq 0, \quad f \neq 0 \text { and } \int_{0}^{1} f d t \leq 4 ;
$$

see, e.g., [1], [6]. Moreover the constant 4 of (2) is the best possible in the sense that (2) is not sufficient for the stability of $x^{\prime \prime}+f x=0$ if the 4 is replaced by $4+\epsilon(\epsilon$, a positive constant) [3]. If $\lambda_{0}$ and $\lambda_{1}$ denote respectively the left and right end-points of the first stability interval of (1) then the first two conditions of (2) imply $\lambda_{0}<0$ while all conditions together imply $\lambda_{1}>0$ (and so $\lambda=0$ is interior to the first interval of stability of (1)). Actually the inequality $\lambda_{1}>0$ is implied by the single condition

$$
\int_{0}^{1} f^{+} d t \leq 4, \text { where } f^{+}(t)=\max [0, f(t)]
$$

(see [6]); moreover, the estimate

$$
\lambda_{1}>4-\int_{0}^{1} f^{+} d t=4\left(1-\frac{1}{4} \int_{0}^{1} f^{+} d t\right)
$$

*Received October 28, 1957. 
easily follows. The object of this note is to obtain a "best possible" refinement of (4), namely, if (3) holds, then

$$
\lambda_{1} \geq \pi^{2}\left(1-\frac{1}{4} \int_{0}^{1} f^{+} d t\right)
$$

where the equality holds only if $f \equiv 0$.

It is known that when $\lambda=\lambda_{1}$, Eq. (1) possesses a "half-periodic" solution $x=y(t)$ satisfying $y(t+1)=-y(t)$; see, e.g., [5]. In case $f \equiv 0$, this function is sin $\pi t$ which satisfies $x^{\prime \prime}+\pi^{2} x=0$ (i.e., (1) for $f \equiv 0$ and $\lambda=\lambda_{1}=\pi^{2}$ ); thus the equality of (5) holds in this case. It is clear then that the italicized assertion above can become false if the $\pi^{2}$ of (5) is replaced by $\pi^{2}+\epsilon$, even if $f \not \equiv 0$. That the assertion can become false if the $\frac{1}{4}$ of (5) is replaced by $\frac{1}{4}-\epsilon$ is obvious from an earlier remark concerning the stability criterion furnished by (2).

In order to prove the italicized assertion, let $y(t)$ denote the half-periodic solution of (1) for $\lambda=\lambda_{1}$ considered above, so that $y^{\prime \prime}+\left[\lambda_{1}+f(t)\right] y=0$, and let $a, a+1$ denote two zeros of $y$. A multiplication by $y$ of both sides of the last equation, followed by an integration and an application of the inequality $f \leq f^{+}$, leads to

$$
\lambda_{1} \int_{a}^{a+1} y^{2} d t \geq \int_{a}^{a+1} y^{\prime 2} d t-\int_{a}^{a+1} f^{+} y^{2} d t
$$

Since $2 y(t)=\int_{a}^{t} y^{\prime} d s-\int_{t}^{a+1} y^{\prime} d s$, it follows that $2|y(t)| \leq \int_{a}^{a+1}\left|y^{\prime}\right| d s$ and hence, by the Schwarz inequality, that $4 y^{2}(t) \leq \int_{a}^{a+1} y^{\prime 2} d t$. Consequently

$$
\int_{a}^{a+1} f^{+} y^{2} d t \leq \frac{1}{4} \int_{a}^{a+1} f^{+} d t \int_{a}^{a+1} y^{\prime 2} d t
$$

hence, by (6),

$$
\lambda_{1} \int_{a}^{a+1} y^{2} d t \geq\left(1-\frac{1}{4} \int_{0}^{1} f^{+} d t\right) \int_{a}^{a+1} y^{\prime 2} d t .
$$

In view of (3), which implies $\lambda_{1}>0$, and the Wirtinger inequality $\pi^{2} \int_{a}^{a+1} y^{2} d t \leq \int_{a}^{a+1} y^{\prime 2} d t$, where the equality holds only if $y=$ const. $\sin \pi(t-a)$ (see, e.g., [2], p. 184), relation (7) implies (5) and the proof of the italicized assertion is now complete.

Remark. The referee has pointed out an interesting parallelism between the inequality (5) and an inequality in a recent paper by J. Peetre [4], see pp. 16-17, for the least eigenvalue of an eigenvalue problem for partial differential equations with a Riemann metric.

\section{REFERENCES}

1. G. Borg, On a Liapounoff criterion of stability, Am. J. Math. 71, 67-70 (1949)

2. G. H. Hardy, J. E. Littlewood, G. Polya, Inequalities, Cambridge, 1952

3. E. R. van Kampen and A. Wintner, On an absolute constant in the theory of variational stability, Am. J. Math. 59, 270-274 (1937)

4. J. Peetre, A generalization of Courant's nodal domain theorem, Math. Scand. 5, 15-20 (1957)

5. M. J. O. Strutt, Lamésche, Mathieusche und verwandte Funktionen in Physik und Technik, Berlin, 1932

6. A. Wintner, On the non-existence of conjugate points, Am. J. Math. 73, 368-380 (1951) 